\title{
APPROXIMATION OF SOLUTIONS TO A DELAY EQUATION WITH A RANDOM FORCING TERM AND NON LOCAL CONDITIONS
}

\author{
RENU CHAUDHARY AND DWIJENDRA N. PANDEY
}

Communicated by William McLean

\begin{abstract}
The existence and approximation of a solution to a delay equation with a random forcing term and non local conditions is studied by using a stochastic version of the well-known Banach fixed point theorem and semigroup theory. Moreover, the convergence of Faedo-Galerkin approximations of the solution is shown. An example is given which illustrates the results.
\end{abstract}

1. Introduction. The differential equations that involve randomness in the mathematical description of a given phenomenon are known as stochastic differential equations. Due to randomness, these differential equations can provide more accurate descriptions than the deterministic differential equations. In recent years, stochastic differential equations in both finite and infinite dimensions have attracted much attention in many areas such as physics, population dynamics, electrical engineering, ecology, medicine, biology and other areas of science and engineering, because of their practical applications in these areas. In fact, real phenomena in different fields of science and engineering, and especially in finance, involving stochastic excitations of a Gaussian white noise type have been extensively investigated, both theoretically and experimentally, over a long period of time. Remember that Gaussian white noise-mathematically described as the formal derivative of a Brownian motion process-is a tolerable abstraction and is never a completely faithful representation of a physical noise source.

2010 AMS Mathematics subject classification. Primary 34A08, 34A45, 34G20.

Keywords and phrases. Analytic semigroup, Faedo-Galerkin approximations, Hilbert space, mild solution, delay equation with a random forcing term.

This work was funded by the Ministry of Human Resource and Development, India, grant No. MHR-02-23-200-44.

Received by the editors on February 12, 2015, and in revised form on May 25, 2016.

DOI: $10.1216 /$ JIE-2016-28-4-481 
For various stochastic differential systems, qualitative properties, such as existence, uniqueness, controllability and stability have been studied by many researchers (see $[\mathbf{6 , 2 9 , 3 0 ]}$ and references therein). Although theoretical procedures for determining the solution of a stochastic integro-differential equation have been widely studied by several authors, in most cases these equations are still not solvable in closed form. Therefore, it is important to find approximate solutions in an explicit form or in a form suitable for applications of numerical methods. For more details on stochastic differential equations and applications, we refer to $[\mathbf{1 3}, \mathbf{1 5}, \mathbf{1 6}, \mathbf{2 3}, \mathbf{2 7}]$.

Non local conditions for an unknown function are given by an expression involving the value(s) of this function at regular intervals rather than continuously over the history period. The nonlocal Cauchy problem was first introduced by Byszewski [11]. He has done pioneering work on nonlocal condition problems $[\mathbf{8}, \mathbf{9}, \mathbf{1 0}]$. These types of conditions are of great importance because they are usually more precise for physical measurements than the classical ones. For instance, Deng [14] showed that the diffusion of a small amount of gas in a transparent tube can be described using the nonlocal conditions better than using local conditions. Therefore, non local conditions are useful in the applied sciences and engineering. For more details on non local conditions we refer to $[\mathbf{1}, \mathbf{2}, \mathbf{3}]$ and the references therein.

The theory of the approximation of solutions to stochastic differential equations has been studied by many authors. Maruyama [24] introduced the Cauchy-Maruyama approximation. Barbu [7] studied Picard type methods to find an approximate solution of a stochastic semilinear equation in a Hilbert space. Balasubramaniam and Dauer [6] gave a Caratheodory successive approximate solution to a semilinear stochastic evolution equation with time delays in Hilbert space. The Taylor series approximation has been studied by Jankovic and Ilic [18] for obtaining an approximate solution of a stochastic differential equation. Kovács and Printems [19, 20] studied the weak and strong order convergence of a fully discrete approximation of a linear stochastic evolution equation. Balasubramaniam [5] studied the Faedo-Galerkin approximate solution of a stochastic semilinear integrodifferential equation in Hilbert space.

Our main concern in this paper is to treat the Faedo-Galerkin approximate solution to a delay equation with a random forcing term 
and non local conditions. Faedo-Galerkin approximate solutions for deterministic differential equations have been studied by many authors $[4,25,26]$, but this approach is new to the literature on the study of delay equations with a random forcing term. For recent work on the Faedo-Galerkin approximations of solutions to different differential equations see $[\mathbf{1 2}, \mathbf{2 1}, \mathbf{2 2}]$ and the references therein.

Let $H$ and $K$ be two separable Hilbert spaces, and let $L(K, H)$ be the space of bounded linear operators from $K$ into $H$. For convenience, we will use the same notation $\|\cdot\|$ to denote the norms in $H, K$ and $L(K, H)$, and use $(\cdot, \cdot)$ to denote inner products in $H$ and $K$. To the best of our knowledge, existence and approximation of solutions to delay equations with a random forcing term and non local conditions have not been considered in the literature. Motivated by the above consideration, and based on the semigroup theory of operators and the Banach fixed point theorem, in the present work we shall use FaedoGalerkin approximations to prove the existence of solutions to the following delay equation with a random forcing term and non local conditions:

$$
\begin{aligned}
\frac{d u(t)}{d t}+A u(t)= & f\left(t, u(t), u_{t}\right) \\
& +\int_{0}^{t} a(t-s) g\left(s, u(s), u_{s}\right) d \omega(s), \quad t \in(0, T],
\end{aligned}
$$

subject to the non local condition

$$
\kappa\left(u_{0}\right)=\phi \quad \text { on }[-\tau, 0],
$$

where $u(\cdot)$ is a random variable with values in a separable Hilbert space $H$. The linear operator $A: D(A) \subset H \rightarrow H$ is closed, densely defined, positive definite and self-adjoint, and is assumed to have a pure point spectrum $0<\lambda_{0} \leq \lambda_{1} \leq \cdots$ and corresponding complete orthonormal eigensystem $\left\{\psi_{i}\right\}$, so that $A \psi_{i}=\lambda_{i} \psi_{i}$ and $\left(\psi_{i}, \psi_{j}\right)=\delta_{i j}$, where $\delta_{i j}$ is the Kronecker delta function. These assumptions on $A$ guarantee that $-A$ is the infinitesimal generator of an analytic semigroup $\left\{e^{-t A}: t \geq 0\right\}$ in $H$. The history $u_{t}$ is defined by

$$
u_{t}(\theta)=u(t+\theta) \quad \text { for } \theta \in[-\tau, 0] .
$$

The functions $f$ and $g$ depend on the history of $u$ over the time interval $(t-\tau, t)$ and are required to meet the hypotheses (H4) and 
(H5) in Section 2. The given $K$-valued Wiener process $\{\omega(t): t \geq 0\}$ is assumed to possess a finite trace, nuclear covariance operator $Q \geq 0$ defined on a complete probability space $\left(\Omega, \mu,\left\{\mu_{t}\right\}_{t \geq 0}, \mathbb{P}\right)$. In the nonlocal condition, $\kappa$ is a map defined from $C_{0}$ into $C_{0}$, where $C_{0}$ is the space of all continuous functions from $[-\tau, 0]$ into $H$ endowed with the supremum norm

$$
\|\varphi\|_{0}=\sup _{-\tau \leq \nu \leq 0}\|\varphi(\nu)\| .
$$

The rest of the paper is organized as follows. In Section 2, we provide some necessary notation, spaces and preliminaries which will be used throughout the paper, and reformulate (1.1) as a stochastic integral equation. In Section 3, we use a sequence of projection operators to define a corresponding sequence of approximate integral equations. The existence and uniqueness of the solution to each approximate integral equation is established. Then, the convergence of these solutions to the desired solution of the original integral equation is shown. In Section 4, we consider the Faedo-Galerkin approximate solution and prove the main result concerning the convergence of such an approximation. In Section 5 , we give an example as an application of the proposed theory.

2. Preliminaries and assumptions. Let $\left(\Omega, \mu,\left\{\mu_{t}\right\}_{t \geq 0}, \mathbb{P}\right)$ be a filtered complete probability space satisfying the usual conditions (i.e., the filtration is a right-continuous increasing family and $\mu_{0}$ contains all $\mathbb{P}$-null sets) and $\left\{\mu_{t}\right\}_{t \geq 0} \subset \mu$. A random variable $u(t)$ is a $\mu$-measurable function from $\Omega$ into $H$, and a collection of random variables

$$
S=\{u(t): \Omega \longrightarrow H\}_{t \in[0, T]}
$$

is called a stochastic process. Let $B_{n}(t), n=1,2,3, \ldots$, be a sequence of one-dimensional Brownian motions defined on the probability space, and set

$$
\omega(t)=\sum_{n=1}^{\infty} \sqrt{\widehat{\lambda}_{n}} B_{n}(t) \varsigma_{n}, \quad t \geq 0,
$$

where $\widehat{\lambda}_{n} \geq 0, n=1,2, \ldots$, are nonnegative real numbers and $\left\{\varsigma_{n}\right\}$ is a complete orthonormal basis for $K$. Let $Q \in L(K, K)$ be the operator 
defined by $Q \varsigma_{n}=\widehat{\lambda}_{n} \varsigma_{n}$ with finite trace

$$
\operatorname{Tr}(Q)=\sum_{n=1}^{\infty} \widehat{\lambda}_{n}<\infty .
$$

Then, the above $K$-valued stochastic process $\omega(t)$ is called a $Q$-Wiener process. We assume that $\mu_{t}=\sigma(\omega(s): 0 \leq s \leq t)$ is the $\sigma$-algebra generated by $\omega$ and that $\mu_{t}=\mu$. Let $\phi \in L(K, H)$, and define

$$
\|\phi\|_{Q}^{2}=\operatorname{Tr}\left(\phi Q \phi^{*}\right)=\sum_{n=1}^{\infty}\left\|\sqrt{\widehat{\lambda}_{n}} \phi \varsigma_{n}\right\|^{2} .
$$

If $\|\phi\|_{Q}<\infty$, then $\phi$ is called a $Q$-Hilbert-Schmidt operator. Let $L_{Q}(K, H)$ denote the space of all $Q$-Hilbert-Schmidt operators $\phi: K \rightarrow$ $H$. The completion $L_{Q}(K, H)$ of $L(K, H)$ with respect to the topology induced by the norm $\|\cdot\|_{Q}$, where $\|\phi\|_{Q}^{2}=\langle\phi, \phi\rangle$, is a Hilbert space with the above norm topology. Let $L_{2}(\Omega, \mu, \mathbb{P} ; H) \equiv L_{2}(\Omega ; H)$ denote the space of strongly-measurable, square integrable random variables equipped with the norm

$$
\|u\|_{L_{2}(\Omega, H)}=\left(E\|u\|_{H}^{2}\right)^{1 / 2},
$$

where $E$ is defined as integration with respect to the probability measure $\mathbb{P}$. We recall that, for a stochastic process $\{F(t): \Omega \rightarrow$ $\left.L_{Q}(K, H)\right\}$,

$$
\begin{aligned}
E\left\|\int_{0}^{t} F(s) d \omega(s)\right\|^{2} & \leq \int_{0}^{t} E\|F(s)\|_{Q}^{2} d s \\
& \leq \operatorname{Tr}(Q) \int_{0}^{t} E\|F(s)\|^{2} d s
\end{aligned}
$$

see Da Prato and Zabczyk [13, Lemma 7.2].

A subspace of $L_{2}(\Omega, H)$ is given by $L_{2}^{0}(\Omega, H)=\left\{f \in L_{2}(\Omega, H)\right.$ : $f$ is $\mu_{0}$-measurable $\}$. Our assumptions on $A$ imply that $0 \in \rho(-A)$, the resolvent set of $-A$. Then, for $0<\alpha \leq 1$, it is possible to define the fractional power $A^{\alpha}$ as a closed linear operator on its domain $D\left(A^{\alpha}\right)$, being dense in $H$, and we denote the Banach space $D\left(A^{\alpha}\right)$ by $H_{\alpha}$ endowed with the norm

$$
\|u\|_{\alpha}=\left\|A^{\alpha} u\right\|, \quad u \in D\left(A^{\alpha}\right),
$$


which is equivalent to the graph norm of $A^{\alpha}$. For more details on fractional power operators, see Pazy [28, page 69]. For all $t \in[-\tau, T]$, define the Banach space $C_{T}^{\alpha}=C\left([-\tau, T], H_{\alpha}\right)$ endowed with the supremum norm, as

$$
\|u\|_{T, \alpha}=\sup _{t \in[-\tau, T]}\left\|A^{\alpha} u(t)\right\| .
$$

Recall that the time history $u_{t}$ is defined by $u_{t}(\theta)=u(t+\theta)$ for $\theta \in$ $[-\tau, 0]$ and belongs to the phase space $C_{0}^{\alpha}$. The norm $\left\|u_{t}\right\|_{0, \alpha}$ is given by:

$$
\left\|u_{t}\right\|_{0, \alpha}=\sup _{\theta \in[-\tau, 0]}\left\|A^{\alpha} u_{t}(\theta)\right\|, \quad u_{t} \in C_{0}^{\alpha} .
$$

To prove our main results, we assume the following hypotheses.

(H1) $a \in L_{\text {loc }}^{2 p}(0, \infty)$ for some $1<p<\infty$.

(H2) There exists a function $h \in C_{T}^{\alpha}$ such that $\kappa\left(h_{0}\right)=\phi$ for all $t \in[-\tau, 0]$.

(H3) The function $h(t) \in D\left(A^{\alpha}\right)$ for all $t \in[-\tau, 0]$ and $h$ is locally Hölder continuous on $[-\tau, 0]$.

(H4) The map $f:[0, T] \times D\left(A^{\alpha}\right) \times C_{0}^{\alpha} \rightarrow H$ is continuous, and there exists a non-decreasing function $L_{f}:[0, \infty) \rightarrow[0, \infty)$, dependent on $R>0$, such that

$$
\|f(t, u, v)\| \leq L_{f}(t)
$$

and

$$
\left\|f\left(t, u_{1}, v_{1}\right)-f\left(t, u_{2}, v_{2}\right)\right\| \leq L_{f}(t)\left[\left\|u_{1}-u_{2}\right\|_{\alpha}+\left\|v_{1}-v_{2}\right\|_{0, \alpha}\right],
$$

for all $t \in[0, T], u_{1}, u_{2} \in B_{R}\left(D\left(A^{\alpha}\right), h(0)\right)$ and $v_{1}, v_{2} \in$ $B_{R}\left(C_{0}^{\alpha}, h\right)$. Here,

$$
B_{R}\left(Z, z_{0}\right)=\left\{z \in Z:\left\|z-z_{0}\right\|_{Z} \leq R\right\}
$$

for any Banach space $\left(Z,\|\cdot\|_{Z}\right)$ and $z_{0} \in Z$.

(H5) The map $g:[0, T] \times D\left(A^{\alpha}\right) \times C_{0}^{\alpha} \rightarrow L_{Q}(K, H)$ is continuous, and there exists a non-decreasing function $L_{g} \in L_{\text {loc }}^{2 q}(0, \infty)$, dependent on $R>0$, where $1<q<\infty$ and $1 / p+1 / q=1$, such that

$$
\|g(t, u, v)\|_{Q} \leq L_{g}(t)
$$


and

$$
\begin{aligned}
& \left\|g\left(t, u_{1}, v_{1}\right)-g\left(t, u_{2}, v_{2}\right)\right\|_{Q} \leq L_{g}(t)\left[\left\|u_{1}-u_{2}\right\|_{\alpha}+\left\|v_{1}-v_{2}\right\|_{0, \alpha}\right], \\
& \quad \text { for all } t \in[0, T], u_{1}, u_{2} \in B_{R}\left(D\left(A^{\alpha}\right), h(0)\right) \text { and } v_{1}, v_{2} \in \\
& \quad B_{R}\left(C_{0}^{\alpha}, h\right) .
\end{aligned}
$$

Definition 2.1. [5] A stochastic process $\{u(t)\}_{\left[-\tau, T_{0}\right]}, 0<T_{0} \leq T$, is called a mild solution of (1.1)-(1.2) if

(i) $\mathrm{u}(\mathrm{t})$ is measurable and $\mu_{t}$-adapted.

(ii) $\mathrm{u}(\mathrm{t})$ is continuous on $\left[0, T_{0}\right]$ almost surely and satisfies the following stochastic integral equation

$$
\begin{aligned}
u(t)= & e^{-t A} h(0)+\int_{0}^{t} e^{-(t-s) A} f\left(s, u(s), u_{s}\right) d s \\
& +\int_{0}^{t} e^{-(t-s) A}\left(\int_{0}^{s} a(s-\tau) g\left(\tau, u(\tau), u_{\tau}\right) d \omega(\tau)\right) d s,
\end{aligned}
$$

for $t \in\left[0, T_{0}\right]$, with $u(t)=h(t)$ for $t \in[-\tau, 0]$.

The existence of solutions of (1.1) is associated with integral equation (2.1), see Da Prato [13, Theorem 5.4].

3. Existence of approximate solutions. In this section, we consider approximate integral equations to equation (2.1) and establish the existence and uniqueness of their solutions. A solution $u$ of equation (2.1) on $\left[-\tau, T_{0}\right]$ is a function $u$ in the Banach space $C_{\alpha}\left(T_{0}\right)=$ $C\left(\left[-\tau, T_{0}\right], L_{2}\left(\Omega, D\left(A^{\alpha}\right)\right)\right)$ of all continuous functions from $\left[-\tau, T_{0}\right]$ into $L_{2}\left(\Omega, D\left(A^{\alpha}\right)\right)$ endowed with the supremum norm

$$
\|u\|_{C_{\alpha}\left(T_{0}\right)}=\sup _{t \in\left[-\tau, T_{0}\right]}\left\|A^{\alpha} u(t)\right\| .
$$

Lemma 3.1. Suppose $0<\alpha \leq 1$, let $-A$ be the infinitesimal generator of an analytic semigroup $\left\{e^{-t A}: t \geq 0\right\}$ such that $\left\|e^{-t A}\right\| \leq C$ for $t \geq 0$, and assume $0 \in \rho(-A)$. Then,

(i) $D\left(A^{\alpha}\right)$ is a Hilbert space.

(ii) For every $t>0$, the operator $A^{\alpha} e^{-t A}$ is bounded and satisfies

$$
\left\|A e^{-t A}\right\| \leq C t^{-1} \quad \text { and } \quad\left\|A^{\alpha} e^{-t A}\right\| \leq C_{\alpha} t^{-\alpha} .
$$


Proof. See Pazy [28].

We define

$$
\bar{h}(t)= \begin{cases}h(t), & t \in[-\tau, 0], \\ h(0), & t \in\left[0, T_{0}\right],\end{cases}
$$

and let $H_{n}$ denote the finite-dimensional subspace of $H$ spanned by the first $n+1$ orthonormal eigenfunctions $\psi_{0}, \psi_{1}, \ldots, \psi_{n}$ of $A$. The corresponding orthoprojector is denoted by by $P^{n}: H \rightarrow H_{n}$ for $n=$ $0,1,2, \ldots$.

We choose $T_{0} \in(0, T]$ and $R>0$ in such a way that

$$
\sup _{0 \leq t \leq T_{0}} E\left\|A^{\alpha}\left(e^{-t A}-I\right) h(0)\right\|^{2} \leq \frac{R}{6} .
$$

Moreover, for $0<\alpha<1 / 2$,

$$
T_{0}<\left(\frac{R(1-2 \alpha)}{6 C(R) C_{\alpha}^{2}}\right)^{1 /(1-2 \alpha)}
$$

where

$$
C(R)=(1+R)\left(T L_{f}^{2}\left(T_{0}\right)+\operatorname{Tr}(Q)\left\|a^{2}\right\|_{L^{p}\left(0, T_{0}\right)}\left\|L_{g}^{2}\right\|_{L^{q}\left(0, T_{0}\right)}\right) .
$$

We define

$$
f_{n}:[0, T] \times D\left(A^{\alpha}\right) \times C_{0}^{\alpha} \longrightarrow H
$$

and

$$
g_{n}:[0, T] \times D\left(A^{\alpha}\right) \times C_{0}^{\alpha} \longrightarrow L_{Q}(K, H),
$$

respectively, by

$$
f_{n}(t, u, v)=f\left(t, P^{n} u, P^{n} v\right)
$$

and

$$
g_{n}(t, u, v)=g\left(t, P^{n} u, P^{n} v\right),
$$

for all $t \in\left[0, T_{0}\right]$, where $\left(P^{n} v\right)(s)=P^{n}(v(s))$ for all $s \in[-\tau, 0]$. Now, define a map $S_{n}$ on $B_{R}\left(C_{\alpha}\left(T_{0}\right), \bar{h}\right)$ by

$$
\left(S_{n} u\right)(t)=e^{-t A} h(0)+\int_{0}^{t} e^{-(t-s) A} f_{n}\left(s, u(s), u_{s}\right) d s
$$




$$
+\int_{0}^{t} e^{-(t-s) A}\left(\int_{0}^{s} a(s-\tau) g_{n}\left(\tau, u(\tau), u_{\tau}\right) d \omega(\tau)\right) d s,
$$

for $t \in\left[0, T_{0}\right]$, with $\left(S_{n} u\right)(t)=h(t)$ for $t \in[-\tau, 0]$.

Theorem 3.2. Let $\alpha \in(0,1 / 2)$ and the hypotheses $(\mathrm{H} 1)-(\mathrm{H} 5)$ hold. If $h(t) \in L_{2}^{0}\left(\Omega, D\left(A^{\alpha}\right)\right)$ for all $t \in[-\tau, 0]$, then there exists a unique $u_{n} \in$ $B_{R}$ such that $S_{n} u_{n}=u_{n}$ for all $n=0,1,2, \ldots$.

Proof. First, we show that $S_{n}: B_{R}\left(C_{\alpha}\left(T_{0}\right), \bar{h}\right) \rightarrow B_{R}\left(C_{\alpha}\left(T_{0}\right), \bar{h}\right)$. For this, we need to show that the map $t \rightarrow\left(S_{n} u\right)(t)$ is continuous from $\left[-\tau, T_{0}\right]$ into $L_{2}\left(\Omega, D\left(A^{\alpha}\right)\right)$ with respect to the $\|\cdot\|$-norm. Thus, for any $u \in B_{R}\left(C_{\alpha}\left(T_{0}\right), \bar{h}\right)$ and $t_{1}, t_{2} \in[-\tau, 0]$, we have

$$
\left(S_{n} u\right)\left(t_{2}\right)-\left(S_{n} u\right)\left(t_{1}\right)=h\left(t_{2}\right)-h\left(t_{1}\right) .
$$

Since the function $h$ satisfies hypothesis $(\mathrm{H} 2)$, we get the required result. Now, for $t_{1}, t_{2} \in\left(0, T_{0}\right]$ with $t_{1}<t_{2}$,

$$
\left(S_{n} u\right)\left(t_{2}\right)-\left(S_{n} u\right)\left(t_{1}\right)=\mathrm{I}+\mathrm{II}+\mathrm{III}+\mathrm{IV}+\mathrm{V},
$$

where the five terms on the right are

$$
\begin{aligned}
\mathrm{I} & =\left(e^{-t_{2} A}-e^{-t_{1} A}\right) h(0), \\
\mathrm{II} & =\int_{t_{1}}^{t_{2}} e^{-\left(t_{2}-s\right) A} f_{n}\left(s, u(s), u_{s}\right) d s, \\
\mathrm{III} & =\int_{0}^{t_{1}}\left(e^{-\left(t_{2}-s\right) A}-e^{-\left(t_{1}-s\right) A}\right) f_{n}\left(s, u(s), u_{s}\right) d s \\
\mathrm{IV} & =\int_{0}^{t_{1}}\left(e^{-\left(t_{2}-s\right) A}-e^{-\left(t_{1}-s\right) A}\right) \\
& \times \int_{0}^{s} a(s-\tau) g_{n}\left(\tau, u(\tau), u_{\tau}\right) d \omega(\tau) d s,
\end{aligned}
$$

and

$$
\mathrm{V}=\int_{t_{1}}^{t_{2}} e^{-\left(t_{2}-s\right) A} \int_{0}^{s} a(s-\tau) g_{n}\left(\tau, u(\tau), u_{\tau}\right) d \omega(\tau) d s
$$

Thus,

$$
E\left\|\left(S_{n} u\right)\left(t_{2}\right)-\left(S_{n} u\right)\left(t_{1}\right)\right\|_{\alpha}^{2}
$$




$$
\leq 5\left(\|\mathrm{I}\|_{\alpha}^{2}+E\|\mathrm{II}\|_{\alpha}^{2}+E\|\mathrm{III}\|_{\alpha}^{2}+E\|\mathrm{IV}\|_{\alpha}^{2}+E\|\mathrm{~V}\|_{\alpha}^{2}\right) .
$$

Now, Pazy [28, Theorem 6.13 (d)] states that, if $0<\beta \leq 1$ and $u \in D\left(A^{\beta}\right)$, then

$$
\left\|\left(e^{-t A}-I\right) u\right\| \leq C_{\beta} t^{\beta}\left\|A^{\beta} u\right\| .
$$

Thus, if $\beta \in(0,1)$ is such that $0<\alpha+\beta<1$, then for $s, t \in\left(0, T_{0}\right]$, we have

$$
\begin{aligned}
\left\|\left(e^{-t A}-I\right) A^{\alpha} e^{-s A} u\right\| & \leq C_{\beta} t^{\beta}\left\|A^{\alpha+\beta} e^{-s A} u\right\| \\
& \leq C_{\beta} C_{\alpha+\beta} t^{\beta} s^{-(\alpha+\beta)}\|u\|,
\end{aligned}
$$

so

$$
\|\mathrm{I}\|_{\alpha}=\left\|\left(I-e^{-\left(t_{2}-t_{1}\right) A}\right) A^{\alpha} e^{-t_{1} A} h(0)\right\| \leq C_{\alpha}\left(t_{2}-t_{1}\right)^{\alpha}\|h(0)\|_{\alpha}
$$

and

$$
\begin{aligned}
E\|\mathrm{II}\|_{\alpha}^{2} & \leq\left(\int_{t_{1}}^{t_{2}}\left\|A^{\alpha} e^{-\left(t_{2}-s\right) A}\right\| E\left\|f_{n}\left(s, u(s), u_{s}\right)\right\| d s\right)^{2} \\
& \leq\left(t_{2}-t_{1}\right) \int_{t_{1}}^{t_{2}} C_{\alpha}^{2}\left(t_{2}-s\right)^{-2 \alpha} L_{f}(s)^{2} d s \\
& \leq C_{\alpha}^{2} L_{f}\left(t_{2}-t_{1}\right)^{2} \frac{\left(t_{2}-t_{1}\right)^{2(1-\alpha)}}{1-2 \alpha}
\end{aligned}
$$

Next, provided $\alpha+\beta<1 / 2$,

$$
\begin{aligned}
E\|\mathrm{III}\|_{\alpha}^{2} & \leq t_{1} \int_{0}^{t_{1}}\left\|\left(I-e^{-\left(t_{2}-t_{1}\right) A}\right) A^{\alpha} e^{-\left(t_{1}-s\right) A}\right\|^{2} L_{f}(s)^{2} d s \\
& \leq C_{\beta}^{2} C_{\alpha+\beta}^{2}\left(t_{2}-t_{1}\right)^{2 \beta} L_{f}\left(t_{1}\right)^{2} \int_{0}^{t_{1}}\left(t_{1}-s\right)^{-2(\alpha+\beta)} d s \\
& =C_{\beta}^{2} C_{\alpha+\beta}^{2} L_{f}\left(t_{1}\right)^{2}\left(t_{2}-t_{1}\right)^{2 \beta} \frac{t_{1}^{1-2(\alpha+\beta)}}{1-2(\alpha+\beta)},
\end{aligned}
$$

with

$$
\begin{aligned}
E\|\mathrm{IV}\|_{\alpha}^{2} \leq & \int_{0}^{t_{1}}\left\|A^{\alpha}\left(e^{-\left(t_{2}-s\right) A}-e^{-\left(t_{1}-s\right) A}\right)\right\|^{2} \\
& \operatorname{Tr}(Q) \int_{0}^{s} a(s-\tau)^{2} E\left\|g_{n}\left(\tau, u(\tau), u_{\tau}\right)\right\|^{2} d \tau d s \\
\leq & \operatorname{Tr}(Q)\left\|a^{2}\right\|_{L^{p}\left(0, T_{0}\right)}\left\|L_{g}^{2}\right\|_{L^{q}\left(0, T_{0}\right)}
\end{aligned}
$$




$$
\begin{gathered}
\int_{0}^{t_{1}}\left\|\left(e^{-\left(t_{2}-t_{1}\right) A}-I\right) A^{\alpha} e^{-\left(t_{1}-s\right) A}\right\|^{2} d s \\
\leq \operatorname{Tr}(Q)\left\|a^{2}\right\|_{L^{p}\left(0, T_{0}\right)}\left\|L_{g}^{2}\right\|_{L^{q}\left(0, T_{0}\right)} \\
C_{\beta}^{2} C_{\alpha+\beta}^{2}\left(t_{2}-t_{1}\right)^{2 \beta} \frac{t_{1}^{1-2(\alpha+\beta)}}{1-2(\alpha+\beta)},
\end{gathered}
$$

and

$$
\begin{aligned}
E\|V\|_{\alpha}^{2} \leq & \int_{t_{1}}^{t_{2}}\left\|A^{\alpha} e^{-\left(t_{2}-s\right) A}\right\|^{2} \\
& \operatorname{Tr}(Q) \int_{0}^{s} a(s-\tau)^{2} E\left\|g_{n}\left(\tau, u(\tau), u_{\tau}\right)\right\|^{2} d \tau d s \\
\leq & \operatorname{Tr}(Q)\left\|a^{2}\right\|_{L_{p}\left(0, T_{0}\right)}\left\|L_{g}^{2}\right\|_{L_{q}\left(0, T_{0}\right)} C_{\alpha}^{2} \frac{\left(t_{2}-t_{1}\right)^{1-2 \alpha}}{1-2 \alpha}
\end{aligned}
$$

The preceding estimates imply that

$$
E\left\|\left(S_{n} u\right)\left(t_{2}\right)-\left(S_{n} u\right)\left(t_{1}\right)\right\|_{\alpha}^{2}=O\left(\left(t_{2}-t_{1}\right)^{\gamma}\right)
$$

for $\gamma=\min (2 \alpha, 2(1-\alpha), 2 \beta)>0$, so the map $t \mapsto\left(S_{n} u\right)(t)$ is continuous from $\left[-\tau, T_{0}\right]$ into $L_{2}\left(\Omega, D\left(A^{\alpha}\right)\right)$ with respect to the $\|\cdot\|$ norm.

Now, for $t \in[-\tau, 0]$, we have

$$
E\left\|\left(S_{n} u\right)(t)-\bar{h}(t)\right\|_{\alpha}^{2}=0,
$$

and for $t \in\left(0, T_{0}\right]$, we have

$$
\begin{aligned}
& E\left\|\left(S_{n} u\right)(t)-\bar{h}(t)\right\|_{\alpha}^{2} \leq 3\left\|A^{\alpha}\left(e^{-t A}-I\right) h(0)\right\|^{2} \\
& +3 \int_{0}^{t}\left\|A^{\alpha} e^{-(t-s) A}\right\|^{2}\left(T E\left\|f_{n}\left(s, u(s), u_{s}\right)\right\|^{2}\right. \\
& \left.\quad+\operatorname{Tr}(Q) \int_{0}^{s}|a(s-\tau)|^{2} E\left\|g_{n}\left(\tau, u(\tau), u_{\tau}\right)\right\|^{2} d \tau\right) d s
\end{aligned}
$$

Using Lemma 3.1 and equations (3.1)-(3.3), we get

$$
E\left\|\left(S_{n} u\right)(t)-\bar{h}(t)\right\|_{\alpha}^{2} \leq 3\left(\frac{R}{6}+C(R) C_{\alpha}^{2} \frac{T_{0}^{1-2 \alpha}}{1-2 \alpha}\right) \leq R,
$$

showing that $S_{n}: B_{R}\left(C_{\alpha}\left(T_{0}\right), \bar{h}\right) \rightarrow B_{R}\left(C_{\alpha}\left(T_{0}\right), \bar{h}\right)$. 
Let $u, v \in B_{R}\left(C_{\alpha}\left(T_{0}\right), \bar{h}\right)$. If $t \in[-\tau, 0]$, then

$$
E\left\|S_{n} u(t)-S_{n} v(t)\right\|^{2}=0,
$$

whereas, if $t \in\left(0, T_{0}\right]$, then

$$
\begin{aligned}
E \| & \left(S_{n} u\right)(t)-\left(S_{n} v\right)(t)\left\|_{\alpha}^{2} \leq 2 \int_{0}^{t}\right\| A^{\alpha} e^{-(t-s) A} \|^{2} \\
& \left(E\left\|f_{n}\left(s, u(s), u_{s}\right)-f_{n}\left(s, v(s), v_{s}\right)\right\|^{2}\right. \\
& \left.+\operatorname{Tr}(Q) \int_{0}^{s} a(s-\tau)^{2} E\left\|g_{n}\left(\tau, u(\tau), u_{\tau}\right)-g_{n}\left(\tau, v(\tau), v_{\tau}\right)\right\|^{2} d \tau\right) d s \\
\leq & 2\left(T L_{f}^{2}\left(T_{0}\right)+\operatorname{Tr}(Q)\left\|a^{2}\right\|_{L^{p}\left(0, T_{0}\right)}\left\|L_{g}^{2}\right\|_{L^{q}\left(0, T_{0}\right)}\right) \\
& \left(E\|u-v\|_{\alpha}^{2}+E\left\|u_{\tau}-v_{\tau}\right\|_{0, \alpha}^{2}\right) C_{\alpha}^{2} \frac{T_{0}^{1-2 \alpha}}{1-2 \alpha} \\
\leq & \frac{2}{R} C(R) C_{\alpha}^{2} \frac{T_{0}^{1-2 \alpha}}{1-2 \alpha}\left(E\|u-v\|_{\alpha}^{2}+E\left\|u_{\tau}-v_{\tau}\right\|_{0, \alpha}^{2}\right) \\
\leq & \frac{1}{3} E\|u-v\|_{C_{\alpha}(T)}^{2} .
\end{aligned}
$$

Thus, taking the supremum over $t \in\left[-\tau, T_{0}\right]$, we get

$$
\left\|S_{n} u-S_{n} v\right\|_{C_{\alpha}(T)}^{2} \leq \frac{1}{3}\|u-v\|_{C_{\alpha}(T)}^{2} .
$$

Hence, $S_{n}$ is a strict contraction on $B_{R}\left(C_{\alpha}\left(T_{0}\right), \bar{h}\right)$, and, by the Banach fixed point theorem, there exists a unique $u_{n} \in B_{R}\left(C_{\alpha}\left(T_{0}\right), \bar{h}\right)$ such that $S_{n} u_{n}=u_{n}$ or, in other words, $u_{n}$ satisfies the approximate integral equation,

$$
u_{n}(t)=\left\{\begin{array}{lr}
h(t), & t \in[-\tau, 0] \\
e^{-t A} h(0)+\int_{0}^{t} e^{-(t-s) A} f_{n}\left(s, u_{n}(s),\left(u_{n}\right)_{s}\right) d s & \\
+\int_{0}^{t} e^{-(t-s) A} \int_{0}^{s} a(s-\tau) g_{n}\left(\tau, u_{n}(\tau),\left(u_{n}\right)_{\tau}\right) d \omega(\tau) d s, & t \in\left[0, T_{0}\right] .
\end{array}\right.
$$

This completes the proof.

Lemma 3.3. Let $h(t) \in L_{2}(\Omega, D(A))$ for all $t \in[-\tau, 0]$. Then $u_{n}(t) \in L_{2}\left(\Omega, D\left(A^{\beta}\right)\right)$ for all $t \in\left[-\tau, T_{0}\right]$ and $0 \leq \beta<1 / 2$. 
Proof. For $t \in[-\tau, 0]$, it is obvious. Therefore, let $t \in(0, T]$. From Theorem 3.2, we have the existence of a unique $u_{n} \in B_{R}\left(C_{\alpha}\left(T_{0}\right), \bar{h}\right)$ satisfying (3.9), and a result of Pazy [28, Theorem 2.6.13 (a)] implies that $e^{-t A}: H \rightarrow L_{2}\left(\Omega, D\left(A^{\beta}\right)\right)$ for $t>0$ and $0 \leq \beta<1 / 2$, and that $D\left(A^{\beta}\right) \subseteq D\left(A^{\alpha}\right)$ for $0 \leq \alpha \leq \beta<1$. By another result of Pazy [28, Theorem 1.2.4], we have $e^{-t A} u \in L_{2}(\Omega, D(A))$ if $u \in L_{2}(\Omega, D(A))$. The required result follows from these facts and the fact that $L_{2}(\Omega, D(A)) \subseteq$ $L_{2}\left(\Omega, D\left(A^{\beta}\right)\right)$ for $0 \leq \beta<1 / 2$.

Lemma 3.4. Let $h(t) \in L_{2}^{0}\left(\Omega, D\left(A^{\alpha}\right)\right)$ for all $t \in[-\tau, 0]$. Then, for any $t \in\left[-\tau, T_{0}\right]$, there exists a constant $C_{t_{0}}$, independent of $n$, such that

$$
E\left\|u_{n}(t)\right\|_{\beta}^{2} \leq C_{t_{0}} \quad \text { for } 0 \leq \alpha<\beta<\frac{1}{2} \text { and } 0 \leq t_{0} \leq T_{0} .
$$

Proof. Applying $A^{\beta}$ to both sides of approximate integral equation (3.9), it follows [28, Theorem 6.13 (c)] that, for any $t \in[-\tau, 0]$,

$$
E\left\|u_{n}(t)\right\|_{\beta}^{2} \leq E\|h(t)\|_{\beta}^{2} \leq\|h\|_{0, \beta},
$$

and, again for $t \in\left(0, T_{0}\right]$,

$$
\begin{aligned}
E\left\|u_{n}(t)\right\|_{\beta}^{2} \leq & 3\left(\left\|A^{\beta} e^{-t A} h(0)\right\|^{2}+E\left\|\int_{0}^{t} A^{\beta} e^{-(t-s) A} f_{n}\left(s, u(s), u_{s}\right) d s\right\|^{2}\right. \\
& \left.+E\left\|\int_{0}^{t} A^{\beta} e^{-(t-s) A} \int_{0}^{s} a(s-\tau) g_{n}\left(\tau, u(\tau), u_{\tau}\right) d \omega(\tau) d s\right\|_{Q}^{2}\right) \\
\leq & 3\left\|A^{\beta} e^{-t A} h(0)\right\|^{2} \\
& +3 T \int_{0}^{t}\left\|A^{\beta} e^{-(t-s) A}\right\|^{2} E\left\|f_{n}\left(s, u(s), u_{s}\right)\right\|^{2} d s \\
& +3 \operatorname{Tr}(Q) \int_{0}^{t}\left\|A^{\beta} e^{-(t-s) A}\right\|^{2} \\
& \quad\left(\int_{0}^{s} a(s-\tau)^{2} E\left\|g_{n}\left(\tau, u(\tau), u_{\tau}\right)\right\|^{2} d \tau\right) d s \\
\leq & 3\left(C_{\beta}^{2} t_{0}^{-2 \beta} E\|h(0)\|^{2}+C_{\beta}^{2} C(R) \frac{T_{0}^{1-2 \beta}}{1-2 \beta}\right) \leq C_{t_{0}} .
\end{aligned}
$$


Theorem 3.5. If the hypotheses (H1)-(H5) hold, and if $h(t) \in$ $L_{2}(\Omega, D(A))$, then $\left\{u_{n}\right\} \subset C_{\alpha}\left(T_{0}\right)$ is a Cauchy sequence and therefore converges to a function $u \in C_{\alpha}\left(T_{0}\right)$ satisfying equation (2.1).

Proof. We divide the proof into two steps.

Step 1 . Let $m, n, n_{0} \in \mathbb{N}$ be such that $n \geq m \geq n_{0}$ and $n_{0}$ is sufficiently large. For $t \in[-\tau, 0]$, we have

$$
E\left\|u_{n}(t)-u_{m}(t)\right\|_{\alpha}^{2}=E\|h(t)-h(t)\|_{\alpha}^{2}=0,
$$

and, for $t \in\left(0, T_{0}\right]$,

$$
\begin{gathered}
E\left\|u_{n}(t)-u_{m}(t)\right\|_{\alpha}^{2} \leq 2 E \| \int_{0}^{t} A^{\alpha} e^{-(t-s) A}\left[f_{n}\left(s, u_{n}(s),\left(u_{n}\right)_{s}\right)\right. \\
\left.-f_{m}\left(s, u_{m}(s),\left(u_{m}\right)_{s}\right)\right] d s \|^{2} \\
\quad+2 E \| \int_{0}^{t} A^{\alpha} e^{-(t-s) A}\left(\int_{0}^{s} a(s-\tau)\right. \\
\left.\left[g_{n}\left(\tau, u_{n}(\tau),\left(u_{n}\right)_{\tau}\right)-g_{m}\left(\tau, u_{m}(\tau),\left(u_{m}\right)_{\tau}\right)\right] d \omega(\tau)\right) d s \|_{Q}^{2}
\end{gathered}
$$

Now, for $0<t_{0}^{\prime}<t$, we have

$$
\begin{gathered}
E\left\|u_{n}(t)-u_{m}(t)\right\|_{\alpha}^{2} \leq 2\left(\int_{0}^{t_{0}^{\prime}}+\int_{t_{0}^{\prime}}^{t}\right)\left\|A^{\alpha} e^{-(t-s) A}\right\|^{2} \\
\left(T E\left\|f_{n}\left(s, u_{n}(s),\left(u_{n}\right)_{s}\right)-f_{m}\left(s, u_{m}(s),\left(u_{m}\right)_{s}\right)\right\|^{2}\right. \\
+\operatorname{Tr}(Q) \int_{0}^{s} a(s-\tau)^{2} \\
\left.E\left\|g_{n}\left(\tau, u_{n}(\tau),\left(u_{n}\right)_{\tau}\right)-g_{m}\left(\tau, u_{m}(\tau),\left(u_{m}\right)_{\tau}\right)\right\|^{2} d \tau\right) d s
\end{gathered}
$$

The first integral of equation (3.10) is bounded by

$$
\begin{aligned}
\int_{0}^{t_{0}^{\prime}}\left\|A^{\alpha} e^{-(t-s) A}\right\|^{2} & \left(T E \| f_{n}\left(s, u_{n}(s),\left(u_{n}\right)_{s}\right)\right. \\
& -f_{m}\left(s, u_{m}(s),\left(u_{m}\right)_{s}\right) \|^{2}+\operatorname{Tr}(Q) \int_{0}^{s} a(s-\tau)^{2}
\end{aligned}
$$




$$
\begin{aligned}
& \left.E\left\|g_{n}\left(\tau, u_{n}(\tau),\left(u_{n}\right)_{\tau}\right)-g_{m}\left(\tau, u_{m}(\tau),\left(u_{m}\right)_{\tau}\right)\right\|^{2} d \tau\right) d s \\
& \leq 2 C(R) C_{\alpha}^{2}\left(t_{0}-t_{0}^{\prime}\right)^{-2 \alpha} t_{0}^{\prime} .
\end{aligned}
$$

Now,

$$
\begin{aligned}
& E\left\|f_{n}\left(s, u_{n}(s),\left(u_{n}\right)_{s}\right)-f_{m}\left(s, u_{m}(s),\left(u_{m}\right)_{s}\right)\right\|^{2} \\
& \leq E\left\|f_{n}\left(s, u_{n}(s),\left(u_{n}\right)_{s}\right)-f_{n}\left(s, u_{m}(s),\left(u_{m}\right)_{s}\right)\right\|^{2} \\
& \quad+E\left\|f_{n}\left(s, u_{m}(s),\left(u_{m}\right)_{s}\right)-f_{m}\left(s, u_{m}(s),\left(u_{m}\right)_{s}\right)\right\|^{2} \\
& \leq 2 L_{f}^{2}\left(T_{0}\right)\left(E\left\|u_{n}-u_{m}\right\|_{s, \alpha}^{2}+E\left\|\left(P^{n}-P^{m}\right) u_{m}(t)\right\|_{\alpha}^{2}\right),
\end{aligned}
$$

and, for $0<\alpha<\beta<1 / 2$, we have

$$
\begin{aligned}
E\left\|A^{\alpha}\left(P^{n}-P^{m}\right) u_{m}(t)\right\|^{2} & \leq E\left\|A^{\alpha-\beta}\left(P^{n}-P^{m}\right) A^{\beta} u_{m}(t)\right\|^{2} \\
& \leq \lambda_{m}^{-2(\beta-\alpha)} E\left\|A^{\beta} u_{m}(t)\right\|^{2} .
\end{aligned}
$$

Therefore,

$$
\begin{aligned}
E \| f_{n}\left(s, u_{n}(s),\left(u_{n}\right)_{s}\right)- & f_{m}\left(s, u_{m}(s),\left(u_{m}\right)_{s}\right) \|^{2} \\
& \leq 2 L_{f}^{2}\left(T_{0}\right)\left(E\left\|u_{n}-u_{m}\right\|_{s, \alpha}^{2}+C_{t_{0}} \lambda_{m}^{-2(\beta-\alpha)}\right) .
\end{aligned}
$$

Similarly,

$$
\begin{aligned}
E \| g_{n}\left(s, u_{n}(s),\left(u_{n}\right)_{s}\right)- & g_{m}\left(s, u_{m}(s),\left(u_{m}\right)_{s}\right) \|^{2} \\
& \leq 2 L_{g}^{2}\left(T_{0}\right)\left(E\left\|u_{n}-u_{m}\right\|_{s, \alpha}^{2}+C_{t_{0}} \lambda_{m}^{-2(\beta-\alpha)}\right) .
\end{aligned}
$$

The second integral of (3.10) is bounded by

$$
\begin{gathered}
\int_{t_{0}^{\prime}}^{t}\left\|A^{\alpha} e^{-(t-s) A}\right\|^{2}\left(T E\left\|f_{n}\left(s, u_{n}(s),\left(u_{n}\right)_{s}\right)-f_{m}\left(s, u_{m}(s),\left(u_{m}\right)_{s}\right)\right\|^{2}\right. \\
+\operatorname{Tr}(Q) \int_{0}^{s} a(s-\tau)^{2} \\
\left.E\left\|g_{n}\left(\tau, u_{n}(\tau),\left(u_{n}\right)_{\tau}\right)-g_{m}\left(\tau, u_{m}(\tau),\left(u_{m}\right)_{\tau}\right)\right\|^{2} d \tau\right) d s \\
\leq 2 \int_{t_{0}^{\prime}}^{t} C_{\alpha}^{2}(t-s)^{-2 \alpha}\left(T L_{f}^{2}\left(T_{0}\right) E\left\|u_{n}-u_{m}\right\|_{s, \alpha}^{2}\right.
\end{gathered}
$$




$$
\begin{gathered}
+L_{f}^{2}\left(T_{0}\right) C_{t_{0}} \lambda_{m}^{-2(\beta-\alpha)}+\operatorname{Tr}(Q) \int_{0}^{s} a(s-\tau)^{2} \\
\left.\left(L_{g}^{2}\left(T_{0}\right) E\left\|u_{n}-u_{m}\right\|_{s, \alpha}^{2}+L_{g}^{2}\left(T_{0}\right) C_{t_{0}} \lambda_{m}^{-2(\beta-\alpha)}\right)\right) d s \\
\leq 2 C(R) C_{\alpha}^{2}\left(\frac{C_{t_{0}}}{\lambda_{m}^{2(\beta-\alpha)}} \frac{T_{0}^{1-2 \alpha}}{(1-2 \alpha)}+\int_{t_{0}^{\prime}}^{t}(t-s)^{-2 \alpha} E\left\|u_{n}-u_{m}\right\|_{\alpha}^{2} d s\right) .
\end{gathered}
$$

Using (3.11) and (3.12) in (3.10), we get

$$
\begin{aligned}
& E\left\|u_{n}(t)-u_{m}(t)\right\|_{\alpha}^{2} \\
& \leq M_{1} t_{0}^{\prime}+M_{2} \lambda_{m}^{-2(\beta-\alpha)}+M_{3} \int_{t_{0}^{\prime}}^{t}(t-s)^{-2 \alpha} E\left\|u_{n}-u_{m}\right\|_{\alpha}^{2} d s
\end{aligned}
$$

where

$$
\begin{aligned}
& M_{1}=\frac{4 C(R) C_{\alpha}^{2}}{\left(t_{0}-t_{0}^{\prime}\right)^{2 \alpha}} \\
& M_{2}=4 C(R) C_{\alpha}^{2} C_{t_{0}} \frac{T_{0}^{1-2 \alpha}}{1-2 \alpha} \\
& M_{3}=4 C(R) C_{\alpha}^{2} .
\end{aligned}
$$

Now replace $t$ by $t+\theta$ in inequality (3.13), where $\theta \in\left[t_{0}^{\prime}-t, 0\right]$, to get

$$
\begin{aligned}
E\left\|u_{n}(t+\theta)-u_{m}(t+\theta)\right\|_{\alpha}^{2} & \leq M_{1} t_{0}^{\prime}+M_{2} \lambda_{m}^{-2(\beta-\alpha)} \\
& +M_{3} \int_{t_{0}^{\prime}}^{t+\theta} \frac{E\left\|u_{n}-u_{m}\right\|_{s, C_{\tilde{h}}}^{2}}{(t+\theta-s)^{2 \alpha}} d s,
\end{aligned}
$$

and then put $s-\theta=\nu$ in (3.14) to obtain

$$
\begin{aligned}
E \| u_{n}(t+\theta) & -u_{m}(t+\theta) \|_{\alpha}^{2} \\
& \leq M_{1} t_{0}^{\prime}+M_{2} \lambda_{m}^{-2(\beta-\alpha)}+M_{3} \int_{t_{0}^{\prime}}^{t} \frac{E\left\|u_{n}-u_{m}\right\|_{\nu, C_{\tilde{h}}}^{2}}{(t-\nu)^{2 \alpha}} d s .
\end{aligned}
$$

Since

$$
\begin{aligned}
\sup _{t_{0}^{\prime}-t \leq \theta \leq 0} E\left\|u_{n}(t+\theta)-u_{m}(t+\theta)\right\|_{\alpha}^{2} & \\
& \leq M_{1} t_{0}^{\prime}+M_{2} \lambda_{m}^{-2(\beta-\alpha)}+M_{3} \int_{t_{0}^{\prime}}^{t} \frac{E\left\|u_{n}-u_{m}\right\|_{\nu, \alpha}^{2}}{(t-\nu)^{2 \alpha}} d s
\end{aligned}
$$


we have

$$
\begin{aligned}
& \sup _{-\tau-t \leq \theta \leq 0} E\left\|u_{n}(t+\theta)-u_{m}(t+\theta)\right\|_{\alpha}^{2} \\
& \leq \sup _{0 \leq \theta+t \leq t_{0}^{\prime}} E\left\|u_{n}(t+\theta)-u_{m}(t+\theta)\right\|_{\alpha}^{2} \\
& \quad+\sup _{t_{0}^{\prime}-t \leq \theta \leq 0} E\left\|u_{n}(t+\theta)-u_{m}(t+\theta)\right\|_{\alpha}^{2} .
\end{aligned}
$$

Using the inequalities above, it follows that

$$
\begin{aligned}
\sup _{-\tau \leq t+\theta \leq t} E\left\|u_{n}(t+\theta)-u_{m}(t+\theta)\right\|_{\alpha}^{2} & \\
\leq\left(M_{1}+M_{4}\right) t_{0}^{\prime}+\left(M_{2}\right. & \left.+M_{5}\right) \lambda_{m}^{-2(\beta-\alpha)} \\
& +M_{3} \int_{t_{0}^{\prime}}^{t} \frac{E\left\|u_{n}-u_{m}\right\|_{\nu, \alpha}^{2}}{(t-\nu)^{2 \alpha}} d s
\end{aligned}
$$

where $M_{4}$ and $M_{5}$ are some constants. Now, applying a generalized Gronwall inequality [17] to the above inequality, and as $t_{0}^{\prime}$ is arbitrarily small, we conclude that $u_{n}$ is a Cauchy sequence.

Step 2. The sequence $u_{n}$ converges to $u$ in $C_{\alpha}\left(T_{0}\right)$.

We have $h(t) \in D(A)$ for all $t \in[-\tau, 0]$. Using Step 1,

$$
\lim _{m \rightarrow \infty} \sup _{\substack{n \geq m \\ 0 \leq t \leq T_{0}}} E\left\|u_{n}(t)-u_{m}(t)\right\|_{\alpha}^{2}=0,
$$

i.e., there exists $u \in C_{\alpha}\left(T_{0}\right)$ such that $u_{n}$ converges to $u$ in the mean square sense. For $u_{n} \in B_{R}\left(C_{\alpha}\left(T_{0}\right), \bar{h}\right)$,

$$
\begin{gathered}
E\left\|u_{n}(t)-u(t)\right\|_{\alpha}^{2} \leq 2 \int_{0}^{t}\left\|A^{\alpha} e^{-(t-s) A}\right\|^{2} \\
\left(T E\left\|f_{n}\left(s, u_{n}(s),\left(u_{n}\right)_{s}\right)-f\left(s, u(s), u_{s}\right)\right\|^{2}\right. \\
+\operatorname{Tr}(Q) \int_{0}^{s}|a(s-\tau)|^{2} \\
\left.E\left\|g_{n}\left(\tau, u_{n}(\tau),\left(u_{n}\right)_{\tau}\right)-g\left(\tau, u(\tau), u_{\tau}\right)\right\|^{2} d \tau\right) d s \\
\leq 2 C_{\alpha}^{2}\left(T E\left\|f\left(t, P^{n} u_{n}(t), P^{n}\left(u_{n}\right)_{t}\right)-f\left(t, u(t), u_{t}\right)\right\|^{2}\right.
\end{gathered}
$$




$$
\begin{gathered}
+\operatorname{Tr}(Q) \int_{0}^{t}|a(t-\tau)|^{2} \\
\left.E\left\|g\left(\tau, P^{n} u_{n}(\tau), P^{n}\left(u_{n}\right)_{\tau}\right)-g\left(\tau, u(\tau), u_{\tau}\right)\right\|^{2} d \tau\right) \frac{T_{0}^{1-2 \alpha}}{1-2 \alpha} \\
\leq \frac{2 C_{\alpha}^{2} T_{0}^{1-2 \alpha}}{1-2 \alpha}\left(T E\left\|f\left(t, P^{n} u_{n}(t), P^{n}\left(u_{n}\right)_{t}\right)-f\left(t, P^{n} u(t), P^{n} u_{t}\right)\right\|_{\alpha}^{2}\right. \\
+T E\left\|f\left(t, P^{n} u(t), P^{n} u_{t}\right)-f\left(t, u(t), u_{t}\right)\right\|_{\alpha}^{2} \\
+\operatorname{Tr}(Q) \int_{0}^{t}|a(t-\tau)|^{2} \\
{\left[E\left\|g\left(\tau, P^{n} u_{n}(\tau), P^{n}\left(u_{n}\right)_{\tau}\right)-g\left(\tau, P^{n} u(\tau), P^{n} u_{\tau}\right)\right\|_{\alpha}^{2}\right.} \\
\left.\left.+E\left\|g\left(\tau, P^{n} u(\tau), P^{n} u_{\tau}\right)-g\left(\tau, u(\tau), u_{\tau}\right)\right\|_{\alpha}^{2}\right] d \tau\right) \\
\leq \frac{2 C_{\alpha}^{2} T_{0}^{1-2 \alpha}}{1-2 \alpha} C(R)\left(E\left\|u_{n}(t)-u_{t}\right\|_{\alpha}^{2}+E\left\|\left(P^{n}-I\right) u(t)\right\|_{\alpha}^{2}\right) .
\end{gathered}
$$

Taking the supremum over $t \in\left[0, T_{0}\right]$, we get

$$
\lim _{n \rightarrow 0} \sup _{0 \leq t \leq T_{0}} E\left\|u_{n}(t)-u(t)\right\|_{\alpha}^{2}=0
$$

in the mean square sense. Let $h(t) \in L_{2}\left(\Omega, D\left(A^{\alpha}\right)\right)$ for $t \in[-\tau, 0]$. Since, for $0<t \leq T_{0}$, the sequence $A^{\alpha} u_{n}(t)$ converges to $A^{\alpha} u(t)$ as $n \rightarrow \infty$, and since $u_{n}(t)=u(t)=h(t)$ for all $n$ when $t \in[-\tau, 0]$, if $-\tau=t=T$, then $A^{\alpha} u_{n}(t)$ converges to $A^{\alpha} u(t)$ in the mean square sense in $L_{2}(\Omega, H)$. Since $u_{n} \in B_{R}\left(C_{\alpha}\left(T_{0}\right), \widetilde{h}\right)$, it follows that $u \in B_{R}\left(C_{\alpha}\left(T_{0}\right), \widetilde{\phi}\right)$ and, for any $0<t_{0} \leq T$,

$$
\lim _{n \rightarrow \infty} \sup _{t_{0} \leq t \leq T_{0}} E\left\|u_{n}(t)-u(t)\right\|_{\alpha}^{2}=0
$$

in the mean square sense. Also, we have

$$
\begin{aligned}
& \sup _{t_{0} \leq t \leq T_{0}} \| f_{n}(\left.t,\left(u_{n}\right)(t),\left(u_{n}\right)_{t}\right)-f\left(t, u(t), u_{t}\right) \|^{2} \\
& \leq L_{f}^{2}\left(T_{0}\right)\left(E\left\|u_{n}-u\right\|_{T_{0}, \alpha}^{2}+E\left\|\left(P^{n}-I\right) u\right\|_{T_{0}, \alpha}^{2}\right) \longrightarrow 0
\end{aligned}
$$

as $n \rightarrow \infty$, and

$$
\sup _{t_{0} \leq t \leq T_{0}}\left\|g_{n}\left(t,\left(u_{n}\right)(t),\left(u_{n}\right)_{t}\right)-g\left(t, u(t), u_{t}\right)\right\|^{2}
$$




$$
\leq L_{g}^{2}\left(T_{0}\right)\left(E\left\|u_{n}-u\right\|_{T_{0}, \alpha}^{2}+E\left\|\left(P^{n}-I\right) u\right\|_{T_{0}, \alpha}^{2}\right) \longrightarrow 0 .
$$

Now we will show that $u$ satisfies the integral equation (2.1). For 0 $<t_{0}<t,(3.9)$ can be written as

$$
\begin{aligned}
u_{n}(t)=e^{-t A} h(0)+ & \left(\int_{0}^{t_{0}}+\int_{t_{0}}^{t}\right) e^{-(t-s) A}\left(f_{n}\left(s, u_{n}(s),\left(u_{n}\right)_{s}\right)\right. \\
& \left.+\int_{0}^{s} a(s-\tau) g_{n}\left(\tau, u_{n}(\tau),\left(u_{n}\right)_{\tau}\right) d \omega(\tau)\right) d s .
\end{aligned}
$$

Thus,

$$
\begin{aligned}
E \| u_{n}(t)-e^{-t A} h(0)-\int_{t_{0}}^{t} e^{-(t-s) A}\left(f_{n}\left(s, u_{n}(s),\left(u_{n}\right)_{s}\right)\right. \\
\left.\quad+\int_{0}^{s} a(s-\tau) g_{n}\left(\tau, u_{n}(\tau),\left(u_{n}\right)_{\tau}\right) d \omega(\tau)\right) d s \|^{2} \\
=E \| \int_{0}^{t_{0}} e^{-(t-s) A}\left(f_{n}\left(s, u_{n}(s),\left(u_{n}\right)_{s}\right)\right. \\
\left.\quad+\int_{0}^{s} a(s-\tau) g_{n}\left(\tau, u_{n}(\tau),\left(u_{n}\right)_{\tau}\right) d \omega(\tau)\right) d s \|^{2} \\
\leq \int_{0}^{t_{0}}\left\|e^{-(t-s) A}\right\|^{2}\left(T E\left\|f_{n}\left(s, u_{n}(s),\left(u_{n}\right)_{s}\right)\right\|^{2}\right. \\
\left.\quad+\operatorname{Tr}(Q) \int_{0}^{s}|a(s-\tau)|^{2} E\left\|g_{n}\left(\tau, u_{n}(\tau),\left(u_{n}\right)_{\tau}\right)\right\|^{2} d \tau\right) d s \\
\leq C^{2} C(R) t_{0} .
\end{aligned}
$$

Sending $n \rightarrow \infty$ in this estimate, we get

$$
\begin{aligned}
E \| u(t)-e^{-t A} h(0)-\int_{t_{0}}^{t} e^{-(t-s) A}\left(f\left(s, u(s), u_{s}\right)\right. & \\
& \left.+\int_{0}^{s} a(s-\tau) g\left(\tau, u(\tau), u_{\tau}\right) d \omega(\tau)\right) d s \|^{2} \leq C^{2} C(R) t_{0} .
\end{aligned}
$$

Since $t_{0}$ is arbitrary, it follows that $u$ satisfies the integral equation (2.1).

Now we will show the uniqueness of $u$. Let $u_{1}$ and $u_{2}$ be two functions satisfying (2.1), and put $u=u_{1}-u_{2}$. Then $u=0$ on $[-\tau, 0]$, and, 
for $t \in\left[0, t_{0}\right]$, we have

$$
E\|u(t)\|_{\alpha}^{2} \leq 2 C(R) C_{\alpha}^{2} \int_{0}^{t}(t-s)^{-2 \alpha} \sup _{-\tau \leq \theta \leq 0} E\|u(s+\theta)\|_{\alpha}^{2} d s,
$$

where $R:=\max \left\{E\left\|u_{1}-\bar{h}\right\|_{C_{\alpha}(T)}^{2}, E\left\|u_{2}-\bar{h}\right\|_{C_{\alpha}(T)}^{2}\right\}$. Let $\bar{\theta} \in[-t, 0]$ and $t \in\left[0, t_{0}\right]$, and assume that $t_{0} \leq \tau$; hence, $0 \leq t \leq \tau$. For $t \leq-\bar{\theta}$, we have $u(t+\bar{\theta})=0$, and, for $t \geq-\bar{\theta}$, we have

$$
\begin{aligned}
& E\|u(t+\bar{\theta})\|_{\alpha}^{2} \leq 2 C(R) C_{\alpha}^{2} \int_{0}^{t+\bar{\theta}}(t+\bar{\theta}-s)^{-2 \alpha} \\
& \cdot \sup _{-\tau \leq \theta \leq 0} E\|u(s+\theta)\|_{\alpha}^{2} d s .
\end{aligned}
$$

We put $\bar{\theta}-s=-\gamma$ in the above inequality to obtain

$$
\begin{aligned}
E\|u(t+\bar{\theta})\|_{\alpha}^{2} \leq & 2 C(R) C_{\alpha}^{2} \int_{-\bar{\theta}}^{t}(t-\gamma)^{-2 \alpha} \\
& \cdot \sup _{-\tau \leq \theta \leq 0} E\|u(\gamma+\theta+\bar{\theta})\|_{\alpha}^{2} d \gamma,
\end{aligned}
$$

and then $\theta=\eta-\bar{\theta}$ to obtain

$$
\begin{aligned}
E\|u(t+\bar{\theta})\|_{\alpha}^{2} \leq & 2 C(R) C_{\alpha}^{2} \int_{-\bar{\theta}}^{t}(t-\gamma)^{-2 \alpha} \\
\cdot & \sup _{-\tau+\bar{\theta} \leq \eta \leq 0} E\|u(\gamma+\eta)\|_{\alpha}^{2} d \gamma .
\end{aligned}
$$

Since $u(\gamma+\eta)=0$ on $[-\tau+\bar{\theta},-\tau]$, the above inequality can be written as

$$
\begin{aligned}
E\left\|u_{t}(\bar{\theta})\right\|_{\alpha}^{2} & \leq 2 C(R) C_{\alpha}^{2} \int_{-\bar{\theta}}^{t}(t-\gamma)^{-2 \alpha} \sup _{-\tau \leq \eta \leq 0} E\left\|u_{\gamma}(\eta)\right\|_{\alpha}^{2} d \gamma \\
& \leq 2 C(R) C_{\alpha}^{2} \int_{0}^{t}(t-\gamma)^{-2 \alpha} \sup _{-\tau \leq \eta \leq 0} E\left\|u_{\gamma}(\eta)\right\|_{\alpha}^{2} d \gamma .
\end{aligned}
$$

Taking the supremum over $\bar{\theta} \in[-\tau, 0]$, we get

$$
E\left\|u_{t}\right\|_{0, \alpha}^{2} \leq 2 C(R) C_{\alpha}^{2} \int_{0}^{t}(t-\gamma)^{-2 \alpha} E\left\|u_{\gamma}\right\|_{0, \alpha}^{2} d \gamma,
$$

and the required result follows by applying the generalized Gronwall's inequality [17]. 
4. Faedo-Galerkin approximations. From the previous sections we know that, for any $0 \leq T_{0} \leq T$, we have a unique $u \in C_{\alpha}\left(T_{0}\right)$ satisfying the integral equation

$$
\begin{aligned}
u(t)= & e^{-t A} h(0)+\int_{0}^{t} e^{-(t-s) A} f\left(s, u(s), u_{s}\right) d s \\
& +\int_{0}^{t} e^{-(t-s) A}\left(\int_{0}^{s} a(s-\tau) g\left(\tau, u(\tau), u_{\tau}\right) d \omega(\tau)\right) d s
\end{aligned}
$$

for $t \in\left[0, T_{0}\right]$, with $u(t)=h(t)$ for $t \in[-\tau, 0]$. Also, there is a unique solution $u_{n} \in C_{\alpha}\left(T_{0}\right)$ of the approximate integral equation

$$
\begin{gathered}
u_{n}(t)=e^{-t A} h(0)+\int_{0}^{t} e^{-(t-s) A} f\left(s, P^{n} u_{n}(s), P^{n}\left(u_{n}\right)_{s}\right) d s \\
+\int_{0}^{t} e^{-(t-s) A}\left(\int_{0}^{s} a(s-\tau) g\left(\tau, P^{n} u_{n}(\tau), P^{n}\left(u_{n}\right) \tau\right) d \omega(\tau)\right) d s
\end{gathered}
$$

for $t \in\left[0, T_{0}\right]$, with $u_{n}(t)=h(t)$ for $t \in[-\tau, 0]$. Now, if we project (4.2) onto $H_{n}$, we get the Faedo-Galerkin approximations $\widehat{u}_{n}=P^{n} u_{n}$ satisfying

$$
\begin{gathered}
\widehat{u}_{n}(t)=e^{-t A} P^{n} h(0)+\int_{0}^{t} e^{-(t-s) A} f\left(s, P^{n} \widehat{u}_{n}(s), P^{n}\left(\widehat{u}_{n}\right)_{s}\right) d s \\
+\int_{0}^{t} e^{-(t-s) A}\left(\int _ { 0 } ^ { s } a ( s - \tau ) g \left(\tau, P^{n} \widehat{u}_{n}(\tau),\right.\right. \\
\left.\left.P^{n}\left(\widehat{u}_{n}\right) \tau\right) d \omega(\tau)\right) d s
\end{gathered}
$$

for $t \in\left[0, T_{0}\right]$, with $\widehat{u}_{n}(t)=P^{n} h(t)$ for $t \in[-\tau, 0]$. The solutions $u(t)$ to $(4.1)$ and $\widehat{u}_{n}(t)$ to $(4.3)$ have the representations

$$
\begin{aligned}
u(t) & =\sum_{i=0}^{\infty} \alpha_{i}(t) u_{i}, & \alpha_{i}(t) & =\left(u(t), u_{i}\right), \\
\widehat{u}_{n}(t) & =\sum_{i=0}^{n} \alpha_{i}^{n}(t) u_{i}, & \alpha_{i}^{n}(t) & =\left(\widehat{u}_{n}(t), u_{i}\right) .
\end{aligned}
$$

As a consequence of Theorems 3.2 and 3.5, we have the following result.

Theorem 4.1. Let the hypotheses (H1)-(H5) hold and assume that $h(t) \in L_{2}(\Omega, D(A))$ for all $t \in[-\tau, 0]$. Then there exists a unique func- 
tion $\widehat{u}_{n} \in C\left(\left[-\tau, T_{0}\right] ; H_{n}\right)$ and a unique $u \in C\left(\left[-\tau, T_{0}\right] ; H\right)$ satisfying (4.3) and (4.1), respectively, such that $\widehat{u}_{n} \rightarrow u$ as $n \rightarrow \infty$.

Theorem 4.2. Let the hypotheses (H1)-(H5) hold. Then we have the following:

(i) If $h(t) \in L_{2}^{0}\left(\Omega, D\left(A^{\alpha}\right)\right)$, then, for any $0<t_{0} \leq T_{0}$,

$$
\lim _{n \rightarrow \infty} \sup _{t_{0} \leq t \leq T_{0}} \sum_{i=0}^{n} \lambda_{i}^{2 \alpha} E\left\|\alpha_{i}(t)-\alpha_{i}^{n}(t)\right\|^{2}=0 .
$$

(ii) If $h(t) \in L_{2}^{0}(\Omega, D(A))$, then

$$
\lim _{n \rightarrow \infty} \sup _{0 \leq t \leq T_{0}} \sum_{i=0}^{n} \lambda_{i}^{2 \alpha} E\left\|\alpha_{i}(t)-\alpha_{i}^{n}(t)\right\|^{2}=0 .
$$

Proof. Since

$$
\begin{aligned}
E\left\|A^{\alpha}\left(u(t)-\widehat{u}_{n}(t)\right)\right\|^{2} & =\sum_{i=0}^{\infty} E\left\|A^{\alpha}\left(\alpha_{i}(t)-\alpha_{i}^{n}(t)\right) u_{i}\right\|^{2} \\
& =\sum_{i=0}^{\infty} \lambda_{i}^{2 \alpha} E\left\|\left(\alpha_{i}(t)-\alpha_{i}^{n}(t)\right) u_{i}\right\|^{2}
\end{aligned}
$$

it follows that

$$
E\left\|A^{\alpha}\left(u(t)-\widehat{u}_{n}(t)\right)\right\|^{2} \geq \sum_{i=0}^{n} \lambda_{i}^{2 \alpha} E\left\|\left(\alpha_{i}(t)-\alpha_{i}^{n}(t)\right) u_{i}\right\|^{2} .
$$

Hence, as a consequence of Theorem 4.1, we get the required result.

5. An example. Let $H=L^{2}(0,1), 0<T<\infty$ and $\tau>0$. Consider the partial differential equation

$$
\begin{aligned}
\frac{\partial u}{\partial t}(x, t)= & \frac{\partial^{2}}{\partial x^{2}} u(t, x)+F(t, x, u) \\
& +\int_{0}^{t} a(t-s) G(s, x, u) d \omega(s), \\
& \int_{-\tau}^{0} r_{3}(-\theta) h_{5}\left(u(\theta, x), \partial_{x} u(\theta, x)\right) d \theta=\phi(x),
\end{aligned}
$$




$$
\begin{aligned}
& u(t, 0)=u(t, 1)=0, \text { where } 0<x<1 \text { and } 0<t<T, \text { with } \\
& F(t, x, u)=f_{1}(t, x)+\int_{0}^{1} h_{1}\left(u(t, y), \partial_{y} u(t, y)\right) d y \\
& \qquad \int_{-\tau}^{0} r_{1}(-\theta) h_{2}\left(u(t+\theta, x), \partial_{x} u(t+\theta, x)\right) d \theta,
\end{aligned}
$$

and

$$
\begin{aligned}
G(t, x, u)=f_{2}(t, x)+ & \int_{0}^{1} h_{3}\left(u(t, y), \partial_{y} u(t, y)\right) d y \\
& \int_{-\tau}^{0} r_{2}(-\theta) h_{4}\left(u(t+\theta, x), \partial_{x} u(t+\theta, x)\right) d \theta .
\end{aligned}
$$

Here, $h_{1}, h_{2}, h_{3}, h_{4}, h_{5}, f_{1}$ and $f_{2}$ are smooth, real-valued functions. Furthermore, $r_{1}, r_{2}, r_{3} \in L^{2}(0,1), a \in L_{\mathrm{loc}}^{2 q}(0, \infty)$, and $\omega$ is a standard $L^{2}(0,1)$-valued Wiener process.

We define the operator $A$ as

$$
A u=-\frac{\partial^{2} u}{\partial x^{2}},
$$

with domain $D(A)=H^{2}(0,1) \cap H_{0}^{1}(0,1)$. Clearly, $A$ is self-adjoint with compact resolvent and is the infinitesimal generator of an analytic semigroup $e^{-t A}$. Moreover, $A$ has a discrete spectrum with the eigenvalues $k^{2} \pi^{2}$ for $k \in \mathbb{N}$, whose corresponding (normalized) eigenfunctions are $e_{k}(x)=\sqrt{2} \sin k \pi x$. Therefore, for $u \in D(A)$,

$$
u(x)=\sum_{k \in \mathbb{N}}\left(u, e_{k}\right) e_{k}(x) .
$$

Now, for $\alpha=1 / 4, D\left(A^{1 / 4}\right)$ (denoted by $H_{1 / 4}$ ) is a Banach space endowed with the norm

$$
\|u\|_{1 / 4}=\left\|A^{1 / 4} u\right\| \quad \text { for } u \in D\left(A^{1 / 4}\right) .
$$

Also, we define the space

$$
C_{t}^{1 / 4}=C\left([-\tau, t], D\left(A^{1 / 4}\right)\right), \quad t \in[0, T]
$$

endowed with the supremum norm

$$
\|\varphi\|_{t, 1 / 4}=\sup _{-\tau \leq \nu \leq t}\|\varphi(\nu)\|_{\alpha}, \quad \varphi \in C_{t}^{1 / 4}
$$


then,

$$
A^{1 / 4} u(x)=\sum_{k \in \mathbb{N}}\left(k^{2} \pi^{2}\right)^{1 / 4}\left\langle u(x), e_{k}(x)\right\rangle e_{k}(x)
$$

with $u \in D\left(A^{1 / 4}\right)$. Differential equation (5.1) can be reformulated as

$$
\begin{aligned}
\frac{d u(t)}{d t}+A u(t)= & F\left(t, u(t), u_{t}\right) \\
& +\int_{0}^{1} a(t-s) G\left(s, u(s), u_{s}\right) d \omega(s), \\
\kappa\left(u_{0}\right)= & \phi \quad \text { on }[-\tau, 0],
\end{aligned}
$$

where $u(t)(x)=u(t, x)$ and $u_{t}(\theta)(x)=u(t+\theta, x)$ for $t \in[0, T]$, $\theta \in[-\tau, 0]$ and $x \in(0,1)$, and where the operator $A$ is defined as in equation (5.2). The functions $F$ and $G$ are defined on $[0, T] \times D\left(A^{\alpha}\right) \times$ $C_{0}^{1 / 2}$ by

$$
\begin{aligned}
F(t, \varphi, \xi)(x)= & f_{1}(t, x)+\int_{0}^{1} h_{1}\left(\varphi(x), \varphi^{\prime}(x)\right) d x \\
& \times \int_{-\tau}^{0} r_{1}(-\theta) h_{2}\left(\xi(\theta)(x), \partial_{x} \xi(\theta)(x)\right) d \theta
\end{aligned}
$$

and

$$
\begin{aligned}
G(t, \varphi, \xi)(x)=f_{2}(t, x)+ & \int_{0}^{1} h_{3}\left(\varphi(x), \varphi^{\prime}(x)\right) d x \\
& \int_{-\tau}^{0} r_{2}(-\theta) h_{4}\left(\xi(\theta)(x), \partial_{x} \xi(\theta)(x)\right) d \theta,
\end{aligned}
$$

where $h_{1}, h_{2}, h_{3}$ and $h_{4}$ are non-decreasing, Lipschitz continuous functions such that

$$
\left\|h_{i}\left(x_{i}, y_{i}\right)-h_{i}\left(x_{i+1}, y_{i+1}\right)\right\| \leq L_{i}\left(\left\|x_{i}-y_{i}\right\|+\left\|x_{i+1}-y_{i+1}\right\|\right)
$$

for $i=1,3$, and

$$
\left\|h_{i}\left(x_{i}, y_{i}\right)-h_{i}\left(x_{i+1}, y_{i+1}\right)\right\| \leq L_{i}\left(\left\|x_{i}-y_{i}\right\|_{[0, \alpha]}+\left\|x_{i+1}-y_{i+1}\right\|_{[0, \alpha]}\right)
$$

for $i=2,4$. The mapping $\kappa: C_{0} \rightarrow C_{0}$ is defined by

$$
\kappa(\varphi)(x)=\int_{-\tau}^{0} r_{3}(-\theta) h_{5}\left(\varphi(\theta, x), \partial_{x} \varphi(\theta, x)\right) d \theta
$$


where the functions $f_{1}, f_{2}:[0, T] \times(0,1) \rightarrow \mathbb{R}$ satisfy $f_{1}(0, \cdot), f_{2}(0, \cdot) \in$ $L^{2}(0,1)$, and there exists $\ell_{i} \in L^{2}(0,1)$ such that

$$
\left\|f_{i}(t, x)-f_{i}(s, x)\right\| \leq \ell_{i}(x)\|t-s\|^{\theta} \quad \text { for } i=1,2 .
$$

Therefore, the functions $F$ and $G$ satisfy

$$
\begin{aligned}
\left\|F\left(t, u_{1}, v_{1}\right)-F\left(t, u_{2}, v_{2}\right)\right\| & \leq L_{f}\left(\left\|u_{1}-u_{2}\right\|_{\alpha}+\left\|v_{1}-v_{2}\right\|_{0, \alpha}\right), \\
\left\|G\left(t, u_{1}, v_{1}\right)-G\left(t, u_{2}, v_{2}\right)\right\| & \leq L_{g}\left(\left\|u_{1}-u_{2}\right\|_{\alpha}+\left\|v_{1}-v_{2}\right\|_{0, \alpha}\right) .
\end{aligned}
$$

Thus assumptions (H1)-(H5) are satisfied and therefore, by the results established in the earlier sections, one can obtain approximate solutions and their convergence.

Acknowledgments. The authors are grateful to the referees and the editor for their helpful remarks.

\section{REFERENCES}

1. D. Bahuguna and S. Agarwal, Approximations of solutions to neutral functional differential equations with nonlocal history conditions, J. Math. Anal. Appl. $\mathbf{3 1 7}$ (2006), 583-602.

2. D. Bahuguna and M. Muslim, Approximation of solutions to non-local history-valued retarded differential equations, Appl. Math. Comp. 174 (2006), 165179 .

3. , A study of nonlocal history-valued retarded differential equations using analytic semigroups, Nonlin. Dynam. Syst. Th. 6 (2006), 63-75.

4. D. Bahuguna, S.K. Srivastava and S. Singh, Approximations of solutions to semilinear integrodifferential equations, Numer. Funct. Anal. Optim. 22 (2001), 487-504.

5. P. Balasubramaniam, M. Syed Ali and J.H. Kim, Faedo-Galerkin approximate solutions for stochastic semilinear integrodifferential equations, Comp. Math. Appl. 58 (2009), 48-57.

6. P. Balasubramaniam and J.P. Dauer, Controllability of semilinear stochastic delay evolution equations in Hilbert spaces, Int. J. Math. Math. Sci. 31 (2002), 157-166.

7. D. Barbu and G. Bocsan, Approximations to mild solutions of stochastic semilinear equations with non-Lipschitz coefficient, Czechoslovak Math. J. 52 (2002), 87-95.

8. L. Byszewski, Theorems about the existence and uniquness of solutions of a semilinear evolution nonlocal Cauchy problem, J. Math. Anal. Appl. 162 (1991), 494-505.

9. Existence and uniqueness of solutions of semilinear evolution nonlocal Cauchy problem, Z. Nauk. Pol. 18 (1993), 109-112. 
10. L. Byszewski and H. Akca, Existence of solutions of a nonlinear functional differential evolution nonlocal problem, Nonlin. Anal. 4 (1998), 65-72.

11. L. Byszewski and V. Lakshmikantham, Theorem about the existence and uniqueness of a solution of a nonlocal abstract Cauchy problem in a Banach space, Appl. Anal. 40 (1990), 11-19.

12. A. Chaddha and D.N. Pandey, Approximations of solutions for a Sobolev type fractional order differential equation, Nonlin. Dynam. Syst. Th. 14 (2014), $11-29$.

13. G. Da Prato and J. Zabczyk, Stochastic equations in infinite dimensions, in Encyclopedia of mathematics and its applications 44, Cambridge University Press, Cambridge, 1992.

14. K. Deng, Exponential decay of solutions of semilinear parabolic equations with nonlocal initial conditions, J. Math. Anal. Appl. 179 (1993), 630-637.

15. L.C. Evans, An introduction to stochastic differential equations, American Mathematical Society, Philadelphia, 2013.

16. T.C. Gard, Introduction to stochastic differential equations, in Mono. Text. Pure Appl. Math. 114, Dekker, New York, 1988.

17. Y. Haiping, G. Jianming and D. Yongsheng, A generalized Gronwall inequality and its application to a fractional differential equation, J. Math. Anal. Appl. 328 (2007), 1075-1081.

18. S. Jankovic and D. Ilic, An analytic approximation of solutions of stochastic differential equations, Comp. Math. Appl. 47 (2004), 903-912.

19. M. Kovács and J. Printems, Weak convergence of a fully discrete of a linear stochastic evolution equation with a positive-type memory term, J. Math. Anal. Appl. 413 (2014), 939-952.

20. - Strong order of convergence of a fully discrete aproximation of a linear stochastic Volterra type evolution equation, Math. Comp. 83 (2014), 23252346 .

21. P. Kumar, D.N. Pandey and D. Bahuguna, Existence of piecewise continuous mild solutions for impulsive functional differential equations with iterated deviating arguments, Elec. J. Diff. Eqn. 241 (2013), 1-15.

22. A A Aproximation of solutions to a fractional differential equation with a deviating argument, Diff. Eqn. Dynam. Syst. 22 (2014), 333-352.

23. X.R. Mao, Stochastic differential equations and applications, Horwood, Chichester, UK, 1997.

24. G. Maruyama, Continuous Markov processes and stochastic equations, Rend. Circ. Mat. Palermo 4 (1955), 48-90.

25. P.D. Miletta, Approximation of solutions to evolution equations, Math. Meth. Appl. Sci. 17 (1994), 753-763.

26. M. Muslim and A.K. Nandakumaran, Existence and approximations of solutions to some fractional order functional integral equations, J. Integral Equations Appl. 22 (2010), 95-114.

27. B. Oksendal, Stochastic differential equations, Springer, Berlin, 2002. 
28. A. Pazy, Semigroups of linear operators and applications to partial differential equations, Appl. Math. Sci. 44, Springer, New York, 1983.

29. Z. Yan and F. Lu, On approximate controllability of fractional stochastic neutral integro-differential inclusions with infinite delay, Appl. Anal. 2014 (2014), 23 pages.

30. Z. Yan and H. Zhang, Asymptotic stability of fractional impulsive neutral stochastic partial integro-differential equations with state-dependent delay, Elec. J. Diff. Eqn. 206 (2013), 1-29.

Department of Mathematics, Indian Institute of Technology Roorkee, ROORKEE 247667, UTTARAKHAND, INDIA

Email address: rrenu94@gmail.com

Department of Mathematics, Indian Institute of Technology Roorkee, RoORKEe 247667, UtTARAKHAND, INDiA

Email address: dwij.iitk@gmail.com 